\title{
Fabrication and optical characterization of silica microspheres
}

$$
\text { Hao Lv }{ }^{1, a^{*}} \text {, Aimei Liü,b }
$$

${ }^{1}$ The Key Laboratory of electric light source and lighting, School of Physics and Electronic information engineering, Hubei Engineering University, Xiaogan 432000, P.R.China

${ }^{2}$ The Key Laboratory of electric light source and lighting, School of Physics and Electronic information engineering, Hubei Engineering University, Xiaogan 432000, P.R.China

a kuerlvhao@hotmail.com, bamlyg2002@aliyun.com

Keywords:Silica; Microspheres; Optical characterization; Fabrication

\begin{abstract}
Recently, microspheres have attracted much attention because of their numerous applications as optical microcavities, for example, in microlasers and micro-sensors, using the excitation of whispering gallery modes. In this paper, an economic and simple method(powders floating method) is used to fabricate silica microspheres The sphere diameters are from $1 \mu \mathrm{m}$ to $10 \mu \mathrm{m}$ by scanning electron microscopy. The optical characterization of the sphere was performed by using the experiment setup of optical characterization
\end{abstract}

\section{Introduction}

In the past few years, microresonators have received a lot of attention ${ }^{[1]}$. Whispering gallery mode (WGM) resonators ${ }^{[2]}$, such as microspheres ${ }^{[3]}$, microtoroids ${ }^{[4]}$ and microrings ${ }^{[5]}$ have been the object of intensive research, both in their fundamental properties (such as quality factors, non-linear effects ${ }^{[6-7]}$ and coupling to quantum systems ${ }^{[8]}$ among many) and applications that include lasers ${ }^{[9-10]}$, chemical $^{[11]}$ and biological ${ }^{[12]}$ sensing and photonic devices ${ }^{[13-14]}$.

Up to now, there have been two primary methods which are used to fabricate silica microspheres. One is the microspheres are fabricated by melting a tip of fiber, but it is difficult at an aspect of the multi-channel coupler because of optical fiber handle; the other is the sol-gel method, but the fabricating process is complex. In this paper, an economic and simple method is used to fabricate silica microspheres and optical characterization of such a sample is researched.

\section{Fabrication}

The apparatus for producing silica microspheres by the method of powders floating method is shown in Fig. 1. The silicon samples were grinded into powders and sieved into a particle size of 500 mesh.

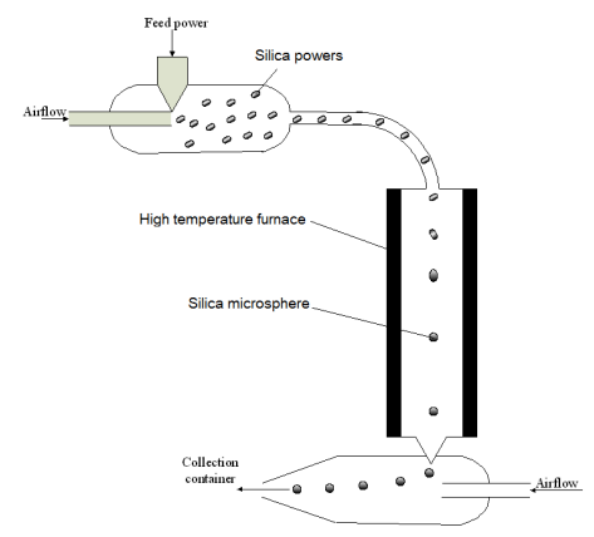

Figure. 1 Process for making silica sphere by the method of powders floating method 
The powers were insufflated into a high temperature furnace $\left(2000^{\circ} \mathrm{C}\right)$ using the high pressure airflow. At the bottom of the high temperature furnace, the high pressure airflow was used. On the one hand, some of the high pressure airflow entered into the high temperature furnace in order to ensure that the silica powers could change into silica microspheres having enough times; on the other hand, the silica microspheres could be collected using high pressure airflow. Fig. 2 is the scanning electron microscope (SEM: Hitachi, S-4700) images of the fabricated silica microspheres.

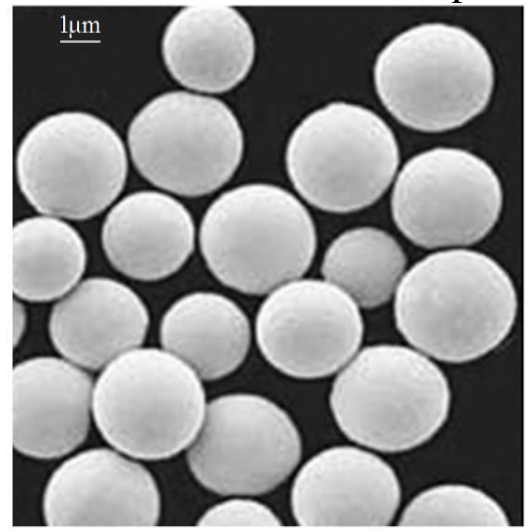

Figure. 2 SEM images of the fabricated silica microspheres

\section{Optical characterization}

We have measured the optical scattering spectrum of a sphere in the attenuated total reflection (ATR) configuration shown in Fig. 3. ATR measurements were performed using a combination of an oil immersion objective $\left[\mathrm{NA}=1.25, \alpha=42-55^{\circ}\right]$ and laser diode (dye laser: $\left.\lambda=570-630 \mathrm{~nm}\right)$. The spot size of the beam on the glass surface is $2 \mu \mathrm{m}$. Scattering intensity was determined using an optical fiber positioned near the sphere.

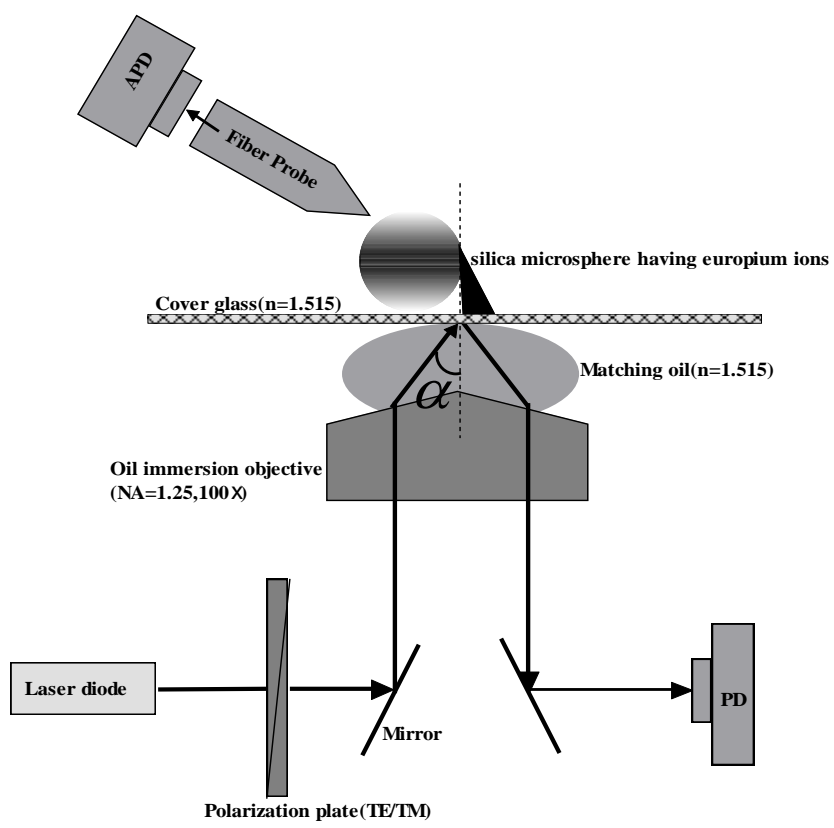

Figure. 3 Setup for WGM excitation experiment using an oil immersion objective by ATR configuration 


\section{Results and discussion}

Fig. 4 and 5 shows the ATR spectra and the scattered light intensity spectra of a single silica microsphere which diameter is $5 \mu \mathrm{m}$ respectively. From the figures, one can see that there are four peaks and four dips whose interval is about $10 \mathrm{~nm}$. The wavelengths of the ATR intensity peaks for the TM-polarized light almost coincide with those for the TE polarized light, as shown in Fig. 4. In Fig.5, the wavelengths of the scattered intensity peaks for the TM-polarized light almost coincide with those of the dips for the TE-polarized light.

These periodic peaks and dips are thought to be related to the excitation of the WGMs in the microsphere ${ }^{[15-16]}$. From Mie scattering theory, the scattered intensity peaks correspond to the excitation wavelengths of the WGMs. The difference in the peak wavelengths comes from different mode numbers of the WGMs. From the full-width at half-maximum of the scattered intensity peak in figure, the quality (Q) factors of the resonances are estimated to be about 100 and 150 for the TM and TE-polarized light respectively.

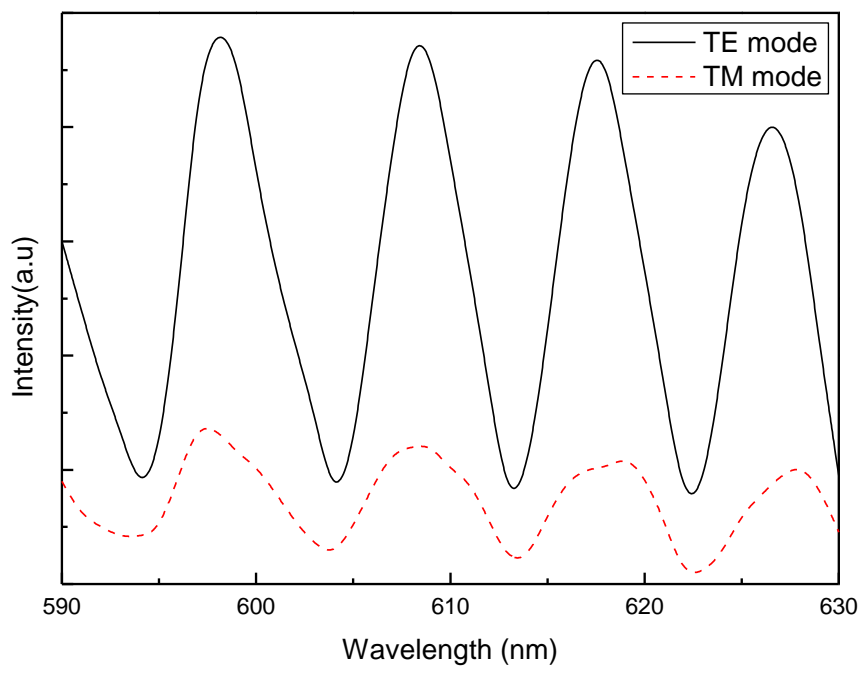

Figure 4 Attenuated total reflection intensity spectra of silica microsphere which diameter is $5 \mu \mathrm{m}$ 


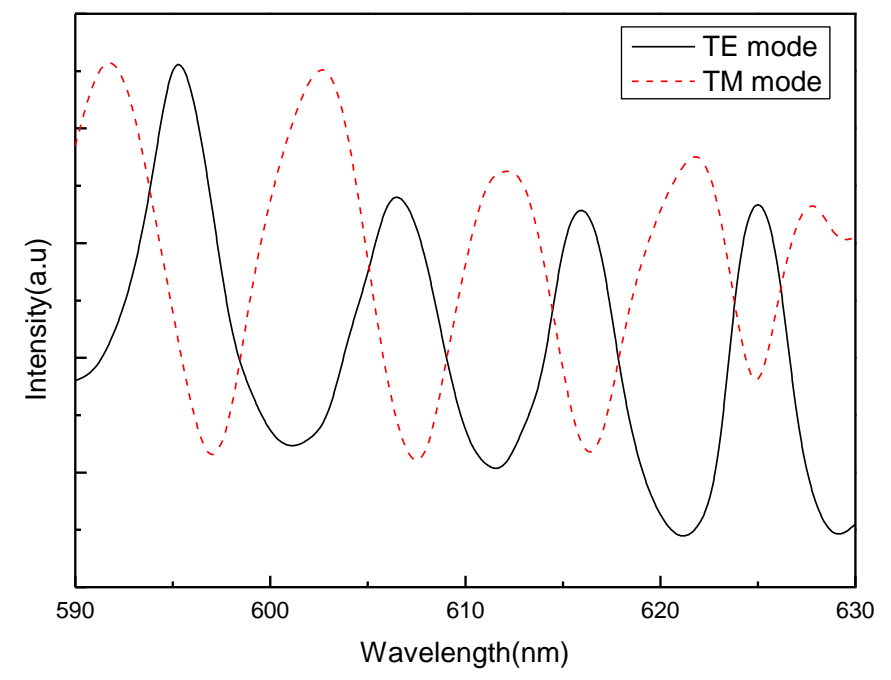

Figure. 5 scattering spectra of silica microsphere which diameter is $5 \mu \mathrm{m}$

\section{Summary}

We have fabricated silica microspheres by using powders floating method. The optical resonances of such a microsphere have been observed by using the experiment setup of optical characterization. The results show that the quality factors of the resonances are estimated to be about 100 and 150 for the TM and TE-polarized light respectively.

\section{References}

[1] K. J. Vahala, Optical microcavities, Nature, 424(2003)839-846

[2]A. B. Matsko and V. S. Ilchenko, Optical Resonators With Whispering-Gallery Modes-Part I: Basics, IEEE J. Sel. Top. Quantum Electron., 12(2006)3-14

[3]S. K.Moon, M. J.Oh, D. H.Paik, T. K.Ryu, K.Park, S.E.Kim, J. H.Park, J. H.Kim, S. W.Choi, A facile method for thepreparation of monodisperse beads with uniform pore sizes for cell culture. Macromol. Rapid Commun., 34（2013） 399-405

[4]D. K. Armani, T. J. Kippenberg, S. M.Spillane, K. J. Vahala, Nature, vol., 421, PP. 925 -929, 2003 [5]A. Melloni, F. Morichetti, M. Martinelli, Polarization conversion in ring resonator phase shifters, Opt. Lett., 29 (2004)2785-2787,

[6]A. E. Fomin, M. L. Gorodetsky, I. S. Gru-dinin, V. S. Ilchenko, Nonstationary nonlinear effects in optical microspheres, JOSA B, 22, (2005)459-465

[7]T. Carmon, L. Yang, K. J. Vahala, Dynamical thermal behavior and thermal self-stability of microcavities, Opt. Express, 12(2004)4742-4750

[8]Y. S. Park, A. K. Cook, H. Wang, Cavity QED with diamond nanocrystals and silica microspheres, Nano. Lett., 6 (2006)2075-2079

[9]M. Cai, O. Painter, K. J. Vahala, Observation of critical coupling in a fiber taper to a silica-microsphere whispering-gallery mode system, Phys. Rev. Lett., 85(2000) 74-77 
[10]S. I. Shopova, G. Farca, A. T. Rosenberger, W. M. Wickramanayake, N. A. Kotov, Appl. Phys. Lett., 85 (2004)6101-6103

[11]J. Zhu, S. K. Ozdemir, Y.F. Xiao, L. Li, L. He, D.-R. Chen, L. Yang, On-chip single nanoparticle detection and sizing by mode splitting in an ultrahigh-Q microresonator, Nat. Photonics 4 (2010) 46-49

[12]M. A. Santiago-Cordoba, S. V. Boriskina, F. Vollmer, M. C. Demirel, Nanoparticle-based protein detection by optical shift of a resonant microcavity, Appl. Phys. Lett., 99(2011)073701

[13]J. Yi, C.Y. Jao, I. L. N. Kandas, B. Liu, Y. Xu, H. D. Robinson, Irreversible adsorption of gold nanospheres on fiber optical tapers and microspheres,Appl. Phys. Lett., 100(2012) 153107

[14]D. K. Armani, T. J. Kippenberg, S.M. Spillane, K. J. Vahala, Ultrahigh-Q toroid microcavity on a chip,” Nature, 421(2003)925-928

[15]M. L. Gorodetsky, V. S. Ilchenko, Optical microsphere resonators: optimal coupling to high-Q whispering-gallery modes, JOSA B, 16(1999) 147-154

[16]A Shinya, M Fukui, Finite-Difference Time-Domain Analysis of the Interaction of Gaussian Evanescent Light with a Single Dielectric Sphere or Ordered Dielectric Spheres, Opt. Rev. 6(1999)215-223 